\title{
CRYSTAL STRUCTURE REPORT
}

$$
\begin{aligned}
& \mathrm{C}_{14} \mathrm{H}_{19} \mathrm{~N}_{3} \\
& 3642-33-1
\end{aligned}
$$

Report prepared for:

Dr. Y. Che - OSI Pharmaceuticals, Inc.

23 April 2002

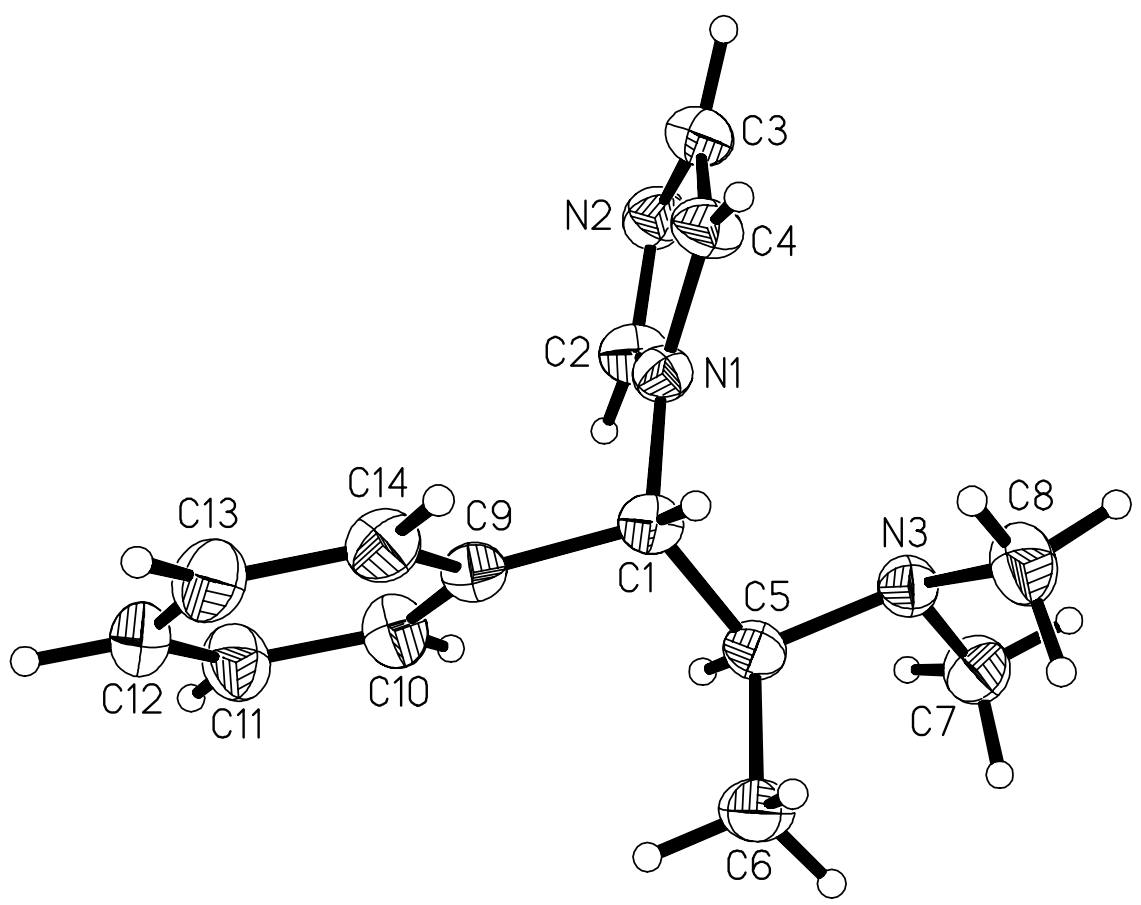

Victor G. Young, Jr.

X-Ray Crystallographic Laboratory

Department of Chemistry

University of Minnesota

207 Pleasant St. S.E.

Minneapolis, MN 55455 


\section{Data collection}

A crystal (approximate dimensions $0.32 \times 0.12 \times 0.10 \mathrm{~mm}^{3}$ ) was placed onto the tip of a $0.1 \mathrm{~mm}$ diameter glass capillary and mounted on a Bruker SMART system for a data collection at 173(2) K. A preliminary set of cell constants was calculated from reflections harvested from three sets of 20 frames. These initial sets of frames were oriented such that orthogonal wedges of reciprocal space were surveyed. This produced initial orientation matrices determined from 57 reflections. The data collection was carried out using MoK $\alpha$ radiation (graphite monochromator) with a frame time of 30 seconds and a detector distance of $4.9 \mathrm{~cm}$. A randomly oriented region of reciprocal space was surveyed to the extent of 2.0 hemispheres and to a resolution of $0.84 \AA$. Four major sections of frames were collected with $0.30^{\circ}$ steps in $\omega$ at 4 different $\phi$ settings and a detector position of $-28^{\circ}$ in $2 \theta$. The intensity data were corrected for absorption and decay (SADABS). ${ }^{1}$ Final cell constants were calculated from 2625 strong reflections from the actual data collection after integration (SAINT 6.01, 1999). ${ }^{2}$ Please refer to Table 1 for additional crystal and refinement information.

Structure solution and refinement

The structure was solved using SHELXS- $86^{3}$ and refined using SHELXL-97. ${ }^{3}$ The space group P2 $2_{1}$ was determined based on systematic absences and intensity statistics. A direct-methods solution was calculated which provided most non-hydrogen atoms from the E-map. Full-matrix least squares / difference Fourier cycles were performed which located the remaining non-hydrogen atoms. All non-hydrogen atoms were refined with anisotropic displacement parameters unless stated otherwise. All hydrogen atoms were placed in ideal positions and refined as riding atoms with relative isotropic displacement parameters. The final full matrix least squares refinement converged to $\mathrm{R} 1=0.0280$ and $\mathrm{wR} 2=0.0735\left(\mathrm{~F}^{2}\right.$, all data $)$.

\section{Structure description}

The structure was found as expected. C5 is $\mathrm{S}$ as known from the synthesis. C1 is $\mathrm{S}$ also. The absolute configuration cannot be determined, but based on chemical knowledge it is correct as presented. The Flack parameter is indeterminate.

Data collection and structure solution were conducted at the X-Ray Crystallographic Laboratory, 160 Kolthoff Hall, Department of Chemistry, University of Minnesota. All calculations were performed using SGI INDY R4400-SC or Pentium computers using the current SHELXTL suite of programs. All publications arising from this report MUST either 1)include Victor G. Young, Jr. as a coauthor or 2)acknowledge Victor G. Young, Jr. and the X-Ray Crystallographic Laboratory. Drafts must be supplied to the coauthor prior to journal submission. Additional drawings will be supplied upon request.

1 An empirical correction for absorption anisotropy, R. Blessing, Acta Cryst. A51, 33 - 38 (1995).

2 SAINT V6.1, Bruker Analytical X-Ray Systems, Madison, WI. 
3 SHELXTL-Plus V5.10, Bruker Analytical X-Ray Systems, Madison, WI. 


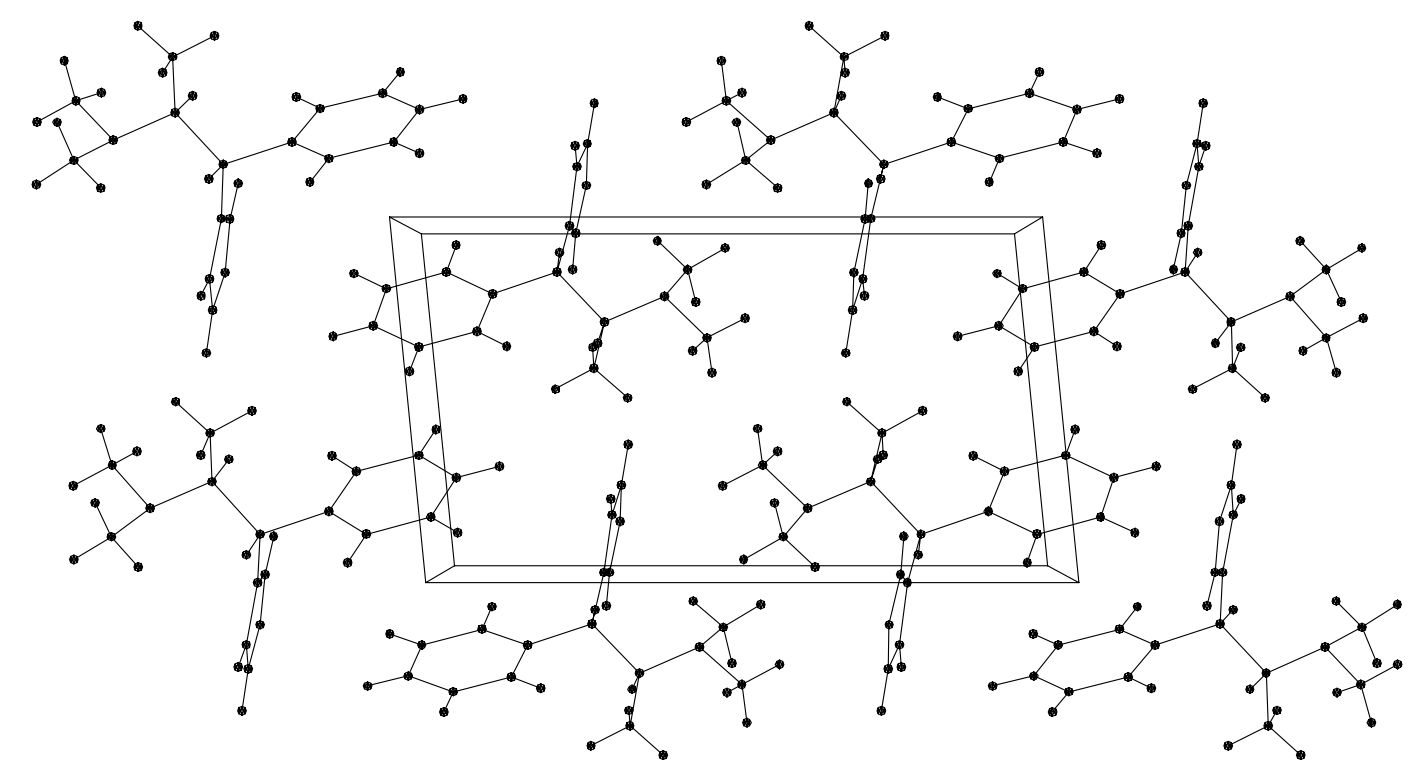


Table 1. Crystal data and structure refinement for $02109 \mathrm{~b}$.

\begin{tabular}{|c|c|c|}
\hline Identification code & \multicolumn{2}{|l|}{$02109 b$} \\
\hline Empirical formula & \multicolumn{2}{|l|}{$\mathrm{C}_{14} \mathrm{H}_{19} \mathrm{~N}_{3}$} \\
\hline Formula weight & \multicolumn{2}{|l|}{229.32} \\
\hline Temperature & \multicolumn{2}{|l|}{$173(2) \mathrm{K}$} \\
\hline Wavelength & \multicolumn{2}{|l|}{$0.71073 \AA$} \\
\hline Crystal system & \multicolumn{2}{|l|}{ Monoclinic } \\
\hline Space group & \multicolumn{2}{|l|}{$\mathrm{P} 2_{1}$} \\
\hline \multirow[t]{3}{*}{ Unit cell dimensions } & $\mathrm{a}=7.908(4) \AA$ & $\alpha=90^{\circ}$ \\
\hline & $\mathrm{b}=5.891(3) \AA$ & $\beta=95.691(8)^{\circ}$. \\
\hline & $c=14.029(7) \AA$ & $\gamma=90^{\circ}$ \\
\hline Volume & \multicolumn{2}{|l|}{$650.2(6) \AA^{3}$} \\
\hline $\mathrm{Z}$ & \multicolumn{2}{|l|}{2} \\
\hline Density (calculated) & \multicolumn{2}{|l|}{$1.171 \mathrm{Mg} / \mathrm{m}^{3}$} \\
\hline Absorption coefficient & \multicolumn{2}{|l|}{$0.071 \mathrm{~mm}^{-1}$} \\
\hline $\mathrm{F}(000)$ & \multicolumn{2}{|l|}{248} \\
\hline Crystal habit and color & \multicolumn{2}{|l|}{ Colorless, Block } \\
\hline Crystal size & \multicolumn{2}{|c|}{$0.32 \times 0.12 \times 0.10 \mathrm{~mm}^{3}$} \\
\hline Theta range for data collection & \multicolumn{2}{|l|}{1.46 to $25.05^{\circ}$} \\
\hline Index ranges & \multicolumn{2}{|c|}{$-9 \leq \mathrm{h} \leq 9,-6 \leq \mathrm{k} \leq 6,0 \leq 1 \leq 16$} \\
\hline Reflections collected & \multicolumn{2}{|l|}{5776} \\
\hline Independent reflections & \multicolumn{2}{|c|}{$2274[\mathrm{R}(\mathrm{int})=0.0197]$} \\
\hline Observed Reflections & \multicolumn{2}{|l|}{2221} \\
\hline Completeness to theta $=25.05^{\circ}$ & \multicolumn{2}{|l|}{$99.8 \%$} \\
\hline Absorption correction & \multicolumn{2}{|c|}{ Semi-empirical from equivalents } \\
\hline Max. and min. transmission & \multicolumn{2}{|c|}{1.000000 and 0.930678} \\
\hline Refinement method & \multicolumn{2}{|c|}{ Full-matrix least-squares on $\mathrm{F}^{2}$} \\
\hline Data / restraints / parameters & \multicolumn{2}{|l|}{2274 / 1 / 157} \\
\hline Goodness-of-fit on $\mathrm{F}^{2}$ & \multicolumn{2}{|l|}{1.048} \\
\hline Final R indices [I>2sigma(I)] & \multicolumn{2}{|c|}{$\mathrm{R} 1=0.0280, \mathrm{wR} 2=0.0728$} \\
\hline $\mathrm{R}$ indices (all data) & \multicolumn{2}{|c|}{$\mathrm{R} 1=0.0289, \mathrm{wR} 2=0.0735$} \\
\hline Absolute structure parameter & \multicolumn{2}{|l|}{$1.0(19)$} \\
\hline Largest diff. peak and hole & \multicolumn{2}{|c|}{0.133 and -0.174 e. $\AA^{-3}$} \\
\hline
\end{tabular}


Table 2. Atomic coordinates (x 10 $\left.0^{4}\right)$ and equivalent isotropic displacement parameters $\left(\AA^{2} \times 10^{3}\right)$ for $02109 \mathrm{~b} . \mathrm{U}_{\mathrm{eq}}$ is defined as one third of the trace of the orthogonalized $U_{\mathrm{ij}}$ tensor.

\begin{tabular}{lrccc}
\hline & $\mathrm{x}$ & $\mathrm{y}$ & $\mathrm{z}$ & $\mathrm{U}(\mathrm{eq})$ \\
\hline $\mathrm{C} 1$ & & & & \\
$\mathrm{~N} 1$ & $8634(2)$ & $6124(2)$ & $2379(1)$ & $26(1)$ \\
$\mathrm{C} 2$ & $10021(1)$ & $4487(2)$ & $2618(1)$ & $26(1)$ \\
$\mathrm{N} 2$ & $9909(2)$ & $2209(2)$ & $2657(1)$ & $33(1)$ \\
C3 & $11384(2)$ & $1218(2)$ & $2894(1)$ & $35(1)$ \\
$\mathrm{C} 4$ & $12504(2)$ & $2994(3)$ & $3011(1)$ & $37(1)$ \\
$\mathrm{C} 5$ & $11696(2)$ & $5007(2)$ & $2846(1)$ & $33(1)$ \\
C6 & $7216(2)$ & $5835(2)$ & $3053(1)$ & $28(1)$ \\
N3 & $5881(2)$ & $7717(3)$ & $2854(1)$ & $38(1)$ \\
C7 & $7946(1)$ & $5639(2)$ & $4051(1)$ & $29(1)$ \\
C8 & $6787(2)$ & $4581(3)$ & $4661(1)$ & $38(1)$ \\
C9 & $8638(2)$ & $7746(3)$ & $4474(1)$ & $41(1)$ \\
C10 & $8017(2)$ & $5970(2)$ & $1316(1)$ & $28(1)$ \\
C11 & $6974(2)$ & $4209(2)$ & $940(1)$ & $36(1)$ \\
C12 & $6536(2)$ & $4030(3)$ & $-39(1)$ & $40(1)$ \\
C13 & $7112(2)$ & $5615(3)$ & $-654(1)$ & $40(1)$ \\
C14 & $8121(2)$ & $7389(3)$ & $-295(1)$ & $41(1)$ \\
& $8576(2)$ & $7563(2)$ & $685(1)$ & $34(1)$ \\
\hline
\end{tabular}


Table 3. Bond lengths $[\AA]$ and angles $\left[{ }^{\circ}\right]$ for $02109 \mathrm{~b}$.

\begin{tabular}{|c|c|c|c|}
\hline C1-N1 & $1.4739(16)$ & N3-C8 & $1.4579(19)$ \\
\hline C1-C9 & $1.5249(18)$ & C7-H7A & 0.9800 \\
\hline $\mathrm{C} 1-\mathrm{C} 5$ & $1.5462(17)$ & C7-H7B & 0.9800 \\
\hline C1-H1A & 1.0000 & $\mathrm{C} 7-\mathrm{H} 7 \mathrm{C}$ & 0.9800 \\
\hline $\mathrm{N} 1-\mathrm{C} 2$ & $1.3460(19)$ & C8-H8A & 0.9800 \\
\hline N1-C4 & $1.3663(17)$ & C8-H8B & 0.9800 \\
\hline $\mathrm{C} 2-\mathrm{N} 2$ & $1.3172(19)$ & C8-H8C & 0.9800 \\
\hline $\mathrm{C} 2-\mathrm{H} 2 \mathrm{~A}$ & 0.9500 & C9-C14 & $1.3917(19)$ \\
\hline $\mathrm{N} 2-\mathrm{C} 3$ & $1.370(2)$ & C9-C10 & $1.3955(19)$ \\
\hline C3-C4 & $1.356(2)$ & C10-C11 & $1.387(2)$ \\
\hline C3-H3A & 0.9500 & $\mathrm{C} 10-\mathrm{H} 10 \mathrm{~A}$ & 0.9500 \\
\hline C4-H4A & 0.9500 & C11-C12 & $1.378(2)$ \\
\hline $\mathrm{C} 5-\mathrm{N} 3$ & $1.4659(17)$ & C11-H11A & 0.9500 \\
\hline C5-C6 & $1.5371(19)$ & $\mathrm{C} 12-\mathrm{C} 13$ & $1.379(2)$ \\
\hline C5-H5A & 1.0000 & $\mathrm{C} 12-\mathrm{H} 12 \mathrm{~A}$ & 0.9500 \\
\hline C6-H6A & 0.9800 & C13-C14 & $1.390(2)$ \\
\hline C6-H6B & 0.9800 & C13-H13A & 0.9500 \\
\hline C6-H6C & 0.9800 & C14-H14A & 0.9500 \\
\hline N3-C7 & $1.4549(17)$ & & \\
\hline $\mathrm{N} 1-\mathrm{C} 1-\mathrm{C} 9$ & $110.02(10)$ & C4-C3-H3A & 124.5 \\
\hline N1-C1-C5 & $110.90(10)$ & N2-C3-H3A & 124.5 \\
\hline $\mathrm{C} 9-\mathrm{C} 1-\mathrm{C} 5$ & $114.22(10)$ & $\mathrm{C} 3-\mathrm{C} 4-\mathrm{N} 1$ & $105.85(12)$ \\
\hline N1-C1-H1A & 107.1 & C3-C4-H4A & 127.1 \\
\hline C9-C1-H1A & 107.1 & N1-C4-H4A & 127.1 \\
\hline C5-C1-H1A & 107.1 & N3-C5-C6 & $115.34(11)$ \\
\hline C2-N1-C4 & $106.27(12)$ & N3-C5-C1 & $110.64(10)$ \\
\hline C2-N1-C1 & $127.68(11)$ & C6-C5-C1 & $109.64(11)$ \\
\hline C4-N1-C1 & $126.05(11)$ & N3-C5-H5A & 106.9 \\
\hline N2-C2-N1 & $113.08(13)$ & C6-C5-H5A & 106.9 \\
\hline $\mathrm{N} 2-\mathrm{C} 2-\mathrm{H} 2 \mathrm{~A}$ & 123.5 & C1-C5-H5A & 106.9 \\
\hline $\mathrm{N} 1-\mathrm{C} 2-\mathrm{H} 2 \mathrm{~A}$ & 123.5 & C5-C6-H6A & 109.5 \\
\hline $\mathrm{C} 2-\mathrm{N} 2-\mathrm{C} 3$ & $103.73(12)$ & C5-C6-H6B & 109.5 \\
\hline $\mathrm{C} 4-\mathrm{C} 3-\mathrm{N} 2$ & $111.07(12)$ & H6A-C6-H6B & 109.5 \\
\hline
\end{tabular}




$\begin{array}{llll}\text { C5-C6-H6C } & 109.5 & \text { C14-C9-C10 } & 118.31(12) \\ \text { H6A-C6-H6C } & 109.5 & \text { C14-C9-C1 } & 119.37(12) \\ \text { H6B-C6-H6C } & 109.5 & \text { C10-C9-C1 } & 122.24(11) \\ \text { C7-N3-C8 } & 111.02(11) & \text { C11-C10-C9 } & 120.72(12) \\ \text { C7-N3-C5 } & 112.60(10) & \text { C11-C10-H10A } & 119.6 \\ \text { C8-N3-C5 } & 114.70(11) & \text { C9-C10-H10A } & 119.6 \\ \text { N3-C7-H7A } & 109.5 & \text { C12-C11-C10 } & 120.15(13) \\ \text { N3-C7-H7B } & 109.5 & \text { C12-C11-H11A } & 119.9 \\ \text { H7A-C7-H7B } & 109.5 & \text { C10-C11-H11A } & 119.9 \\ \text { N3-C7-H7C } & 109.5 & \text { C11-C12-C13 } & 120.00(13) \\ \text { H7A-C7-H7C } & 109.5 & \text { C11-C12-H12A } & 120.0 \\ \text { H7B-C7-H7C } & 109.5 & \text { C13-C12-H12A } & 120.0 \\ \text { N3-C8-H8A } & 109.5 & \text { C12-C13-C14 } & 120.03(13) \\ \text { N3-C8-H8B } & 109.5 & \text { C12-C13-H13A } & 120.0 \\ \text { H8A-C8-H8B } & 109.5 & \text { C14-C13-H13A } & 120.0 \\ \text { N3-C8-H8C } & 109.5 & \text { C13-C14-C9 } & 120.78(13) \\ \text { H8A-C8-H8C } & 109.5 & \text { C13-C14-H14A } & 119.6 \\ \text { H8B-C8-H8C } & 109.5 & \text { C9-C14-H14A } & 119.6\end{array}$

Symmetry transformations used to generate equivalent atoms: 
Table 4. Anisotropic displacement parameters $\left(\AA^{2} \times 10^{3}\right)$ for 02109b. The anisotropic displacement factor exponent takes the form: $-2 \pi^{2}\left[h^{2} a^{* 2} U_{11}+\ldots+2 h k a^{*} b^{*} U_{12}\right]$

\begin{tabular}{lcccccc}
\hline & $\mathrm{U}_{11}$ & $\mathrm{U}_{22}$ & $\mathrm{U}_{33}$ & $\mathrm{U}_{23}$ & $\mathrm{U}_{13}$ & $\mathrm{U}_{12}$ \\
\hline $\mathrm{C} 1$ & $26(1)$ & $21(1)$ & $32(1)$ & $0(1)$ & $2(1)$ & $-2(1)$ \\
$\mathrm{N} 1$ & $24(1)$ & $24(1)$ & $29(1)$ & $-1(1)$ & $4(1)$ & $-3(1)$ \\
$\mathrm{C} 2$ & $30(1)$ & $26(1)$ & $43(1)$ & $1(1)$ & $4(1)$ & $-2(1)$ \\
$\mathrm{N} 2$ & $36(1)$ & $34(1)$ & $35(1)$ & $1(1)$ & $5(1)$ & $4(1)$ \\
$\mathrm{C} 3$ & $28(1)$ & $43(1)$ & $38(1)$ & $-2(1)$ & $-1(1)$ & $2(1)$ \\
$\mathrm{C} 4$ & $25(1)$ & $34(1)$ & $40(1)$ & $-2(1)$ & $-1(1)$ & $-5(1)$ \\
$\mathrm{C} 5$ & $25(1)$ & $27(1)$ & $31(1)$ & $0(1)$ & $2(1)$ & $-3(1)$ \\
$\mathrm{C} 6$ & $29(1)$ & $44(1)$ & $39(1)$ & $3(1)$ & $3(1)$ & $4(1)$ \\
$\mathrm{N} 3$ & $28(1)$ & $32(1)$ & $28(1)$ & $-1(1)$ & $3(1)$ & $-3(1)$ \\
$\mathrm{C} 7$ & $37(1)$ & $44(1)$ & $34(1)$ & $3(1)$ & $7(1)$ & $-4(1)$ \\
$\mathrm{C} 8$ & $45(1)$ & $43(1)$ & $34(1)$ & $-4(1)$ & $1(1)$ & $-10(1)$ \\
$\mathrm{C} 9$ & $26(1)$ & $27(1)$ & $31(1)$ & $1(1)$ & $4(1)$ & $0(1)$ \\
$\mathrm{C} 10$ & $40(1)$ & $34(1)$ & $33(1)$ & $3(1)$ & $3(1)$ & $-7(1)$ \\
$\mathrm{C} 11$ & $42(1)$ & $41(1)$ & $37(1)$ & $-4(1)$ & $0(1)$ & $-8(1)$ \\
$\mathrm{C} 12$ & $40(1)$ & $55(1)$ & $26(1)$ & $-1(1)$ & $5(1)$ & $2(1)$ \\
$\mathrm{C} 13$ & $44(1)$ & $47(1)$ & $35(1)$ & $10(1)$ & $11(1)$ & $-3(1)$ \\
$\mathrm{C} 14$ & $33(1)$ & $33(1)$ & $37(1)$ & $2(1)$ & $8(1)$ & $-5(1)$ \\
& & & & & & \\
\hline
\end{tabular}


Table 5. Hydrogen coordinates ( $\left.\mathrm{x} 10^{4}\right)$ and isotropic displacement parameters $\left(\AA^{2} \times 10^{3}\right)$ for $02109 \mathrm{~b}$.

\begin{tabular}{|c|c|c|c|c|}
\hline & $\mathrm{x}$ & $\mathrm{y}$ & $\mathrm{z}$ & $\mathrm{U}(\mathrm{eq})$ \\
\hline $\mathrm{H} 1 \mathrm{~A}$ & 9124 & 7677 & 2494 & 31 \\
\hline $\mathrm{H} 2 \mathrm{~A}$ & 8873 & 1400 & 2528 & 40 \\
\hline $\mathrm{H} 3 \mathrm{~A}$ & 13690 & 2836 & 3185 & 44 \\
\hline $\mathrm{H} 4 \mathrm{~A}$ & 12192 & 6477 & 2882 & 40 \\
\hline H5A & 6635 & 4362 & 2880 & 33 \\
\hline H6A & 5052 & 7631 & 3328 & 56 \\
\hline H6B & 5298 & 7519 & 2210 & 56 \\
\hline $\mathrm{H} 6 \mathrm{C}$ & 6443 & 9202 & 2898 & 56 \\
\hline H7A & 6440 & 3091 & 4400 & 57 \\
\hline H7B & 5781 & 5544 & 4685 & 57 \\
\hline $\mathrm{H} 7 \mathrm{C}$ & 7358 & 4396 & 5309 & 57 \\
\hline H8A & 9412 & 8426 & 4050 & 61 \\
\hline H8B & 9261 & 7423 & 5098 & 61 \\
\hline $\mathrm{H} 8 \mathrm{C}$ & 7708 & 8803 & 4557 & 61 \\
\hline $\mathrm{H} 10 \mathrm{~A}$ & 6560 & 3121 & 1359 & 43 \\
\hline $\mathrm{H} 11 \mathrm{~A}$ & 5837 & 2813 & -287 & 48 \\
\hline $\mathrm{H} 12 \mathrm{~A}$ & 6814 & 5485 & -1325 & 48 \\
\hline $\mathrm{H} 13 \mathrm{~A}$ & 8506 & 8492 & -718 & 50 \\
\hline $\mathrm{H} 14 \mathrm{~A}$ & 9277 & 8785 & 927 & 41 \\
\hline
\end{tabular}


Table 6. Torsion angles $\left[{ }^{\circ}\right]$ for $02109 \mathrm{~b}$.

\begin{tabular}{lclc}
\hline C9-C1-N1-C2 & $-70.81(16)$ & C1-C5-N3-C7 & $-159.45(12)$ \\
C5-C1-N1-C2 & $56.53(17)$ & C6-C5-N3-C8 & $-52.85(15)$ \\
C9-C1-N1-C4 & $109.20(13)$ & C1-C5-N3-C8 & $72.30(14)$ \\
C5-C1-N1-C4 & $-123.46(13)$ & N1-C1-C9-C14 & $-100.00(14)$ \\
C4-N1-C2-N2 & $0.03(17)$ & C5-C1-C9-C14 & $134.53(12)$ \\
C1-N1-C2-N2 & $-179.96(10)$ & N1-C1-C9-C10 & $76.56(15)$ \\
N1-C2-N2-C3 & $-0.06(16)$ & C5-C1-C9-C10 & $-48.91(17)$ \\
C2-N2-C3-C4 & $0.07(15)$ & C14-C9-C10-C11 & $1.2(2)$ \\
N2-C3-C4-N1 & $-0.06(15)$ & C1-C9-C10-C11 & $-175.35(13)$ \\
C2-N1-C4-C3 & $0.02(15)$ & C9-C10-C11-C12 & $-0.8(2)$ \\
C1-N1-C4-C3 & $-179.99(12)$ & C10-C11-C12-C13 & $-0.3(2)$ \\
N1-C1-C5-N3 & $45.91(14)$ & C11-C12-C13-C14 & $0.8(2)$ \\
C9-C1-C5-N3 & $170.91(11)$ & C12-C13-C14-C9 & $-0.4(2)$ \\
N1-C1-C5-C6 & $174.23(10)$ & C10-C9-C14-C13 & $-0.68(19)$ \\
C9-C1-C5-C6 & $-60.77(14)$ & C1-C9-C14-C13 & $176.01(13)$ \\
C6-C5-N3-C7 & $75.39(15)$ & & \\
& & &
\end{tabular}

Symmetry transformations used to generate equivalent atoms: 